\title{
Dense near octagons with four points on each line, III
}

\author{
Bart De Bruyn
}

Ghent University, Department of Pure Mathematics and Computer Algebra, Krijgslaan 281 (S22), B-9000 Gent, Belgium, E-mail: bdb@cage.ugent. be

\begin{abstract}
This is the third paper dealing with the classification of the dense near octagons of order $(3, t)$. Using the partial classification of the valuations of the possible hexes obtained in [12], we are able to show that almost all such near octagons admit a big hex. Combining this with the results of [11], where we classified the dense near octagons of order $(3, t)$ with a big hex, we get an incomplete classification for the dense near octagons of order $(3, t)$ : there are 28 known examples and a few open cases. For each open case, we have a rather detailed description of the structure of the near octagons involved.
\end{abstract}

Keywords: near polygon, near octagon, generalized quadrangle, valuation MSC2000: 05B25, 51E12

\section{Introduction}

In this introduction, we will only recall the basic notions from the theory of near polygons. In Section 2 we will explain some of the more advanced notions. The reader will also find there the definitions of the near polygons which will occur in the various propositions, theorems and corollaries of this introduction.

A near polygon $\mathcal{S}=(\mathcal{P}, \mathcal{L}, \mathrm{I}), \mathrm{I} \subseteq \mathcal{P} \times \mathcal{L}$, is a partial linear space with the property that for every point $p$ and every line $L$, there exists a unique point on $L$ nearest to $p$. Here distances $\mathrm{d}(\cdot, \cdot)$ are measured in the point graph or collinearity graph $\Gamma$ of $\mathcal{S}$. If $d$ is the diameter of $\Gamma$, then the near polygon is called a near $2 d$-gon. A near 0 -gon is just a point and a near 2 -gon is a line. In the sequel, we will denote the unique line with $s+1$ points by $\mathbb{L}_{s+1}$. 
Near quadrangles are usually called generalized quadrangles (GQ's; Payne and Thas [17]).

A near polygon is said to have order $(s, t)$ if every line is incident with precisely $s+1$ points and if every point is incident with precisely $t+1$ lines. A near polygon is called dense if every line is incident with at least three points and if every two points at distance 2 have at least two common neighbours. By Lemma 19 of Brouwer and Wilbrink [2], every point of a dense near polygon $\mathcal{S}$ is incident with the same number of lines. We denote this number by $t_{\mathcal{S}}+1$. By Theorem 4 of [2], every two points of a dense near polygon at distance $\delta$ from each other are contained in a unique convex sub- $2 \delta$-gon. These convex subpolygons are called quads if $\delta=2$ and hexes if $\delta=3$. The existence of quads was already shown in Proposition 2.5 of Shult and Yanushka [19]. A convex subpolygon $F \neq \mathcal{S}$ of a dense near polygon $\mathcal{S}$ is called big in $\mathcal{S}$ if every point of $\mathcal{S}$ not contained in $F$ is collinear with a (necessarily unique) point of $F$. A convex subpolygon $F$ of a dense near polygon $\mathcal{S}$ is called classical in $\mathcal{S}$ if for every point $x$ of $\mathcal{S}$ there exists a (necessarily unique) point $\pi_{F}(x)$ in $F$ such that $\mathrm{d}(x, y)=\mathrm{d}\left(x, \pi_{F}(x)\right)+$ $\mathrm{d}\left(\pi_{F}(x), y\right)$ for every point $y$ of $F$. Every big subpolygon is classical.

All generalized quadrangles of order $(3, t)$ have been determined by Dixmier and Zara [16], see also Payne and Thas [17, 6.2]. In De Bruyn [4], a partial classification of the dense near hexagons of order $(3, t)$ was obtained. There are 10 known examples and 4 open cases (the so-called exceptional near hexagons of type (I), (II), (III) and (IV)). The present paper is the third one dealing with the classification of the dense near octagons of order $(3, t)$. In De Bruyn [11], we determined "all" dense near octagons of order $(3, t)$ with a big hex:

Proposition 1.1 If $\mathcal{S}$ is a dense near octagon of order $(3, t)$ with a big hex, then $\mathcal{S}$ is isomorphic to either $\mathbb{L}_{4} \times H$ for some exceptional dense near hexagon $H$ of order $(3, t)$, or to one of the near octagons of the following list: $\mathbb{L}_{4} \times \mathbb{L}_{4} \times \mathbb{L}_{4} \times \mathbb{L}_{4}, \mathbb{L}_{4} \times \mathbb{L}_{4} \times W(3), \mathbb{L}_{4} \times \mathbb{L}_{4} \times Q(4,3), \mathbb{L}_{4} \times \mathbb{L}_{4} \times \operatorname{GQ}(3,5)$, $\mathbb{L}_{4} \times \mathbb{L}_{4} \times Q(5,3), \mathbb{L}_{4} \times D W(5,3), \mathbb{L}_{4} \times D Q(6,3), \mathbb{L}_{4} \times D H(5,9), \mathbb{L}_{4} \times$ $(\mathrm{GQ}(3,5) \otimes \mathrm{GQ}(3,5)), \mathbb{L}_{4} \times(Q(5,3) \otimes Q(5,3)), W(3) \times W(3), W(3) \times Q(4,3)$, $W(3) \times \mathrm{GQ}(3,5), W(3) \times Q(5,3), Q(4,3) \times Q(4,3), Q(4,3) \times \mathrm{GQ}(3,5)$, $Q(4,3) \times Q(5,3), \mathrm{GQ}(3,5) \times \mathrm{GQ}(3,5), \mathrm{GQ}(3,5) \times Q(5,3), Q(5,3) \times Q(5,3)$, $D H(5,9) \otimes Q(5,3),(Q(5,3) \otimes Q(5,3)) \otimes_{1} Q(5,3),(Q(5,3) \otimes Q(5,3)) \otimes_{2}$ $Q(5,3),(\mathrm{GQ}(3,5) \otimes \mathrm{GQ}(3,5)) \otimes_{1} \mathrm{GQ}(3,5),(\mathrm{GQ}(3,5) \otimes \mathrm{GQ}(3,5)) \otimes_{2} \mathrm{GQ}(3,5)$, $D W(7,3), D Q(8,3), D H(7,9)$.

In De Bruyn [12], we obtained a partial classification of the valuations of the dense near hexagons of order $(3, t)$. In the present paper, we will use this 
classification to show that almost all dense near octagons of order $(3, t)$ must have a big hex:

Theorem 1.2 Let $\mathcal{S}$ be a dense near octagon of order $(3, t)$ without big hexes. Then all quads are isomorphic to $\mathbb{L}_{4} \times \mathbb{L}_{4}$ and all hexes are isomorphic to $\mathbb{L}_{4} \times \mathbb{L}_{4} \times \mathbb{L}_{4}$ or to an exceptional hex of type $(I V)$.

Proposition 1.1 and Theorem 1.2 give a classification of the dense near octagons of order $(3, t)$ : there are 28 examples and five open cases (four in Proposition 1.1 and one in Theorem 1.2).

It is not know whether there exist near octagons as described in Theorem 1.2. The following result, which is an immediate corollary of De Bruyn [7, Corollary 3] and Theorem 1.2, says something about their structure.

Corollary 1.3 Let $\mathcal{S}$ be a dense near octagon of order $(3, t)$ without big hexes. Then there exist constants $\alpha_{1}$ and $\alpha_{2}$ such that every point is contained in $\alpha_{1}$ hexes isomorphic to $\mathbb{L}_{4} \times \mathbb{L}_{4} \times \mathbb{L}_{4}$ and $\alpha_{2}$ exceptional hexes of type $(I V)$.

In Section 5.1, we will show the following:

Theorem 1.4 (Section 5.1) Let $\mathcal{V}$ denote a class of dense near $2 \delta$-gons, $\delta \geq 2$, and let $\mathcal{S}$ be a dense near $2 d$-gon, $d \geq \delta+1$, satisfying the following property.

For every convex sub- $(2 \delta+2)$-gon $F$ of $\mathcal{S}$, there exists a constant $\alpha_{F}$ such that every point of $F$ is contained in precisely $\alpha_{F}$ convex subpolygons which are contained in $F$ and which are isomorphic to an element of $\mathcal{V}$.

Then there exists a constant $\alpha$ such that every point of $\mathcal{S}$ is contained in precisely $\alpha$ convex subpolygons isomorphic to an element of $\mathcal{V}$.

Combining Theorem 1.4 with the partial classification of the dense near hexagons of order $(3, t)$ (see Proposition 2.4), we immediately obtain the following corollary (see Section 5.2):

Corollary 1.5 Let $\mathcal{S}$ be a dense near polygon of order $(3, t)$ and let $Q$ be one of the five generalized quadrangles of order $(3, t)$. Then there exists a constant $\alpha$ such that every point of $\mathcal{S}$ is contained in $\alpha$ quads isomorphic to $Q$. 
Combining Theorem 1.4 with Proposition 1.1, Theorem 1.2 and Corollary 1.3, we will obtain the following result in Section 5.2.

Corollary 1.6 Let $\mathcal{S}$ be a dense near polygon of order $(3, t)$ and let $H$ be a dense near hexagon of order $(3, t)$ isomorphic to either $W(3) \times \mathbb{L}_{4}, Q(4,3) \times$ $\mathbb{L}_{4}, \operatorname{GQ}(3,5) \times \mathbb{L}_{4}, D W(5,3), D Q(6,3), D H(5,9), \operatorname{GQ}(3,5) \otimes \mathrm{GQ}(3,5)$ or an exceptional near hexagon of type $(I),(I I),(I I I)$ or $(I V)$. Then there exists a constant $\alpha$ such that every point of $\mathcal{S}$ is contained in $\alpha$ hexes isomorphic to $H$.

Similar properties hold for the other near hexagons if we impose extra conditions on the structure of the near polygon.

Corollary 1.7 Let $\mathcal{S}$ be a dense near polygon of order $(3, t)$ which does not contain convex suboctagons isomorphic to $(Q(5,3) \otimes Q(5,3)) \otimes_{2} Q(5,3)$. Then every point of $\mathcal{S}$ is contained in the same number of $\mathbb{L}_{4} \times \mathbb{L}_{4} \times \mathbb{L}_{4}$-hexes, the same number of $Q(5,3) \times \mathbb{L}_{4}$-hexes and the same number of $Q(5,3) \otimes Q(5,3)$ hexes.

\section{More advanced notions and properties}

Suppose $\mathcal{S}$ is a near polygon. As usual, we will denote distances in $\mathcal{S}$ by $\mathrm{d}\left(*_{1}, *_{2}\right)$, where each of $*_{1}, *_{2}$ can be either a point or a nonempty set of points. We denote by $\Gamma_{i}(*)$ the set of points at distance $i$ from $*$, and $\left\langle *_{1}, \ldots, *_{k}\right\rangle$ denotes the smallest convex subspace containing $*_{1}, \ldots, *_{k}$, i.e. the intersection of all convex subspaces containing $*_{1}, \ldots, *_{k}$.

Dense near polygons satisfy several nice properties. Besides the existence of convex subpolygons and the fact that all points are incident with the same number of lines, there are two others which we would like to mention.

Proposition 2.1 ([7, Theorem 1]) Let $\mathcal{S}$ be a dense near 2d-gon. Then there exist constants $n_{i}, i \in\{0, \ldots, d\}$, such that $n_{i}=\left|\Gamma_{i}(x)\right|$ for every $i \in\{0, \ldots, d\}$ and for every point $x$ of $\mathcal{S}$.

Proposition 2.2 ([19, Proposition 2.6]) Let $x$ be a point and $Q$ a quad of a dense near polygon. Then precisely one of the following holds.

(a) There exists a unique point $\pi_{Q}(x)$ in $Q$ nearest to $x$ and $d(x, y)=$ $d\left(x, \pi_{Q}(x)\right)+d\left(\pi_{Q}(x), y\right)$ for every point $y$ of $Q$.

(b) The points in $Q$ nearest to $x$ form an ovoid of $Q$, i.e. a set of points meeting each line in a unique point. 
If case (a) of Proposition 2.2 occurs, then $x$ is called classical with respect to $Q$. If case (b) occurs, then $x$ is called ovoidal with respect to $Q$.

If $K$ and $L$ are two lines of a near polygon, then $N=\mid\{\mathrm{d}(k, l) \mid k \in K, l \in$ $L\} \mid \in\{2,3\}$. If $N=2$, then for every point $k \in K$, there exists a unique point $l \in L$ such that $\mathrm{d}(k, l)=\mathrm{d}(K, L)$. In this case, $K$ and $L$ are called parallel $(K \| L)$.

The maximal and next-to-maximal singular subspaces of a polar space of rank $n \geq 2$ define a near $2 n$-gon, which is called a dual polar space ([3], [19]). Dual polar spaces are also called classical near polygons. We will denote dual polar spaces by putting the letter " $D$ " in front of the name of the corresponding polar spaces. So, $D W(2 n-1, q)$ denotes the dual polar space associated with a symplectic polarity in $\mathrm{PG}(2 n-1, q), D Q(2 n, q)$ denotes the dual polar space associated with a nonsingular parabolic quadric in $\mathrm{PG}(2 n, q)$ and $D H\left(2 n-1, q^{2}\right)$ denotes the dual polar space associated with a nonsingular hermitian variety in $\operatorname{PG}\left(2 n-1, q^{2}\right)$. As in [17, 3.1.1], we will denote the classical generalized quadrangles of order $(3, t)$ by $W(3), Q(4,3)$ and $Q(5,3)$, respectively. We will denote the unique generalized quadrangle of order $(3,5)$ by $\operatorname{GQ}(3,5)$.

If $\mathcal{A}_{1}$ and $\mathcal{A}_{2}$ are two near polygons, then there exists up to isomorphism a unique near polygon $\mathcal{A}_{1} \times \mathcal{A}_{2}$ whose point graph is isomorphic to the cartesian product of the point graphs of $\mathcal{A}_{1}$ and $\mathcal{A}_{2}$. We say that $\mathcal{A}_{1} \times \mathcal{A}_{2}$ is the direct product of $\mathcal{A}_{1}$ and $\mathcal{A}_{2}$.

In [5], a construction was given which can be used to construct so-called glued near polygons of type $\mathcal{A}_{1} \otimes \mathcal{A}_{2}$ from given near polygons $\mathcal{A}_{1}$ and $\mathcal{A}_{2}$ satisfying nice properties. We refer to [5] for the precise definition. For the purpose of this paper, it suffices to know that such a glued near polygon $\mathcal{S}$ satisfies the following properties: (i) there exists a partition $T_{i}, i \in\{1,2\}$, of $\mathcal{S}$ in convex subpolygons isomorphic to $\mathcal{A}_{i}$; (ii) every element of $T_{1}$ intersects every element of $T_{2}$ in a line; (iii) every line of $\mathcal{S}$ is contained in an element of $T_{1} \cup T_{2}$; (iv) for every $i \in\{1,2\}$, for every $F \in T_{i}$ and for all $F_{1}^{\prime}, F_{2}^{\prime} \in T_{3-i}$, the lines $F \cap F_{1}^{\prime}$ and $F \cap F_{2}^{\prime}$ are parallel. For a given near polygon $\mathcal{S}$, let $\Delta_{1}(\mathcal{S})$ denote the set of all unordered pairs $\left\{T_{1}, T_{2}\right\}$ satisfying the abovementioned conditions (i), (ii), (iii) and (iv). If there are up to isomorphism $k$ glued near polygons of type $\mathcal{A}_{1} \otimes \mathcal{A}_{2}$, then we will denote these glued near polygons by $\mathcal{A}_{1} \otimes_{1} \mathcal{A}_{2}, \mathcal{A}_{1} \otimes_{2} \mathcal{A}_{2}, \ldots, \mathcal{A}_{1} \otimes_{k} \mathcal{A}_{2}$. If there is only one glued near polygon of type $\mathcal{A}_{1} \otimes \mathcal{A}_{2}$, then we will denote this glued near polygon by $\mathcal{A}_{1} \otimes \mathcal{A}_{2}$. In Proposition 1.1, $(Q(5,3) \otimes Q(5,3)) \otimes_{1} Q(5,3)$ denotes the unique glued near octagon of type $(Q(5,3) \otimes Q(5,3)) \otimes Q(5,3)$ satisfying the property that the three $Q(5,3)$-quads through any given point have a line in common. Similarly, $(\mathrm{GQ}(3,5) \otimes \mathrm{GQ}(3,5)) \otimes_{1} \mathrm{GQ}(3,5)$ denotes the unique glued near octagon of type $(\mathrm{GQ}(3,5) \otimes \mathrm{GQ}(3,5)) \otimes \mathrm{GQ}(3,5)$ satisfying the 
property that the three $\mathrm{GQ}(3,5)$-quads through any given point have a line in common. (We refer to Sections 3.3 and 3.4 of [11] for more details.)

The classifications of the generalized quadrangles and the dense near hexagons of order $(3, t)$ are given in the following propositions.

Proposition 2.3 ([16]; [17, 6.2]) Every generalized quadrangle of order $(3$, t) is isomorphic to either the $(4 \times 4)$-grid $\mathbb{L}_{4} \times \mathbb{L}_{4}, W(3), Q(4,3), \operatorname{GQ}(3,5)$ or $Q(5,3)$.

Proposition 2.4 (Main Theorem of [4]) Let $\mathcal{S}$ be a dense near hexagon of order $(3, t)$.

(1) If $\mathcal{S}$ is classical or glued, then $\mathcal{S}$ is isomorphic to either $\mathbb{L}_{4} \times \mathbb{L}_{4} \times \mathbb{L}_{4}$, $W(3) \times \mathbb{L}_{4}, Q(4,3) \times \mathbb{L}_{4}, \operatorname{GQ}(3,5) \times \mathbb{L}_{4}, Q(5,3) \times \mathbb{L}_{4}, D W(5,3), D Q(6,3)$, $D H(5,9), \mathrm{GQ}(3,5) \otimes \mathrm{GQ}(3,5)$ or $Q(5,3) \otimes Q(5,3)$.

(2) If $\mathcal{S}$ is neither classical nor glued, then only quads isomorphic to the $(4 \times 4)$-grid or to $Q(4,3)$ occur and none of these quads is big. Moreover, there exist constants $a$ and $b$ such that every point of $\mathcal{S}$ is contained in a grids and $b$ quads isomorphic to $Q(4,3)$. If $v$ denotes the total number of points of $\mathcal{S}$, then $(v, t, a, b)$ is equal to either $(5848,19,160,5),(6736,21,171,10)$, $(8320,27,120,43)$ or $(20608,34,595,0)$.

A non-classical and non-glued dense near hexagon of order $(3, t)$ is called exceptional of type (I), (II), (III), respectively (IV), if $(v, t, a, b)$ is equal to $(5848,19,160,5),(6736,21,171,10),(8320,27,120,43)$, respectively $(20608$, $34,595,0)$.

Let $\mathcal{S}=(\mathcal{P}, \mathcal{L}, \mathrm{I})$ be a dense near polygon. A function $f$ from $\mathcal{P}$ to $\mathbb{N}$ is called a valuation of $f$ if it satisfies the following properties $(f(x)$ is called the value of $x$ ):

$\left(V_{1}\right)$ there exists at least one point with value 0 ;

$\left(V_{2}\right)$ every line $L$ of $\mathcal{S}$ contains a unique point $x_{L}$ with smallest value and $f(x)=f\left(x_{L}\right)+1$ for every point $x$ of $L$ different from $x_{L}$;

$\left(V_{3}\right)$ every point $x$ of $\mathcal{S}$ is contained in a convex subpolygon $F_{x}$ satisfying the following properties:

- $f(y) \leq f(x)$ for every point $y$ of $F_{x}$,

- every point $z$ of $\mathcal{S}$ which is collinear with a point $y$ of $F_{x}$ and which satisfies $f(z)=f(y)-1$ also belongs to $F_{x}$. 
It can be shown, see Proposition 2.5 of [13], that the convex subpolygon $F_{x}$ in Property $\left(V_{3}\right)$ is unique. If $f$ is a valuation, then we denote by $O_{f}$ the set of points with value 0 . A quad of $\mathcal{S}$ is called special if it contains at least two points of $O_{f}$, or equivalently, if $Q \cap O_{f}$ is an ovoid of $Q$ ([13, Corollary 2.11]). Valuations were used in [15] to classify all dense near octagons of order $(2, t)$ and in the present paper, we will use them to obtain a partial classification of the dense near octagons of order $(3, t)$. The reason why valuations are such an important tool for classifying dense near polygons is because of the following proposition, which we take from [13, Proposition 2.6].

Proposition 2.5 Let $\mathcal{S}$ be a dense near $2 n$-gon and let $F$ be a convex subpolygon of $\mathcal{S}$. For every point $x$ of $\mathcal{S}$ and for every point $y$ of $F$, we define $f_{x}(y):=d(x, y)-d(x, F)$. Then $f_{x}$ is a valuation of $F$.

Several classes of valuations were described in [13]. We list the ones which we will need in the present paper.

Examples. (a) Let $x$ denote an arbitrary point of a dense near polygon $\mathcal{S}$. For every point $y$ of $\mathcal{S}$, we define $f(y)=\mathrm{d}(x, y)$. Then $f$ is a so-called classical valuation of $\mathcal{S}$.

(b) Let $O$ be an ovoid of $\mathcal{S}$. For every point $y$ of $\mathcal{S}$, we define $f(y)=0$ if $y \in O$ and $f(y)=1$ otherwise. Then $f$ is a so-called ovoidal valuation of $\mathcal{S}$.

(c) Let $x$ be a point of a dense near $2 n$-gon $\mathcal{S}(n \geq 2)$ and let $O$ be a set of points at distance $n$ from $x$ with the property that every line at distance $n-1$ from $x$ has a unique point in common with $O$. If $y$ is a point of $\mathcal{S}$, then we define $f(y)=\mathrm{d}(x, y)$ if $\mathrm{d}(x, y) \leq n-1, f(y)=n-2$ if $y \in O$ and $f(y)=n-1$ otherwise. Then $f$ is a so-called semi-classical valuation of $\mathcal{S}$.

(d) Let $\mathcal{S}$ be a dense near polygon and let $F$ be a convex subpolygon of $\mathcal{S}$ which is classical in $\mathcal{S}$. Suppose $f^{\prime}$ is a valuation of $F$. For every point $y$ of $\mathcal{S}$, we define $f(y)=\mathrm{d}\left(y, \pi_{F}(y)\right)+f^{\prime}\left(\pi_{F}(y)\right)$. Then $f$ is a valuation of $\mathcal{S}$, which is called the extension of $f^{\prime}$.

(e) The following type of valuation was described in [10]. Let $\mathcal{S}$ be a dense glued near hexagon of type $\mathcal{A}_{1} \otimes \mathcal{A}_{2}$, and let $\left\{T_{1}, T_{2}\right\} \in \Delta_{1}(\mathcal{S})$ such that every quad of $T_{i}, i \in\{1,2\}$, is isomorphic to $\mathcal{A}_{i}$. Suppose $X$ is a set of points of $\mathcal{S}$ satisfying the following properties: (i) $\mathrm{d}\left(x_{1}, x_{2}\right)=2$ for any two distinct points $x_{1}$ and $x_{2}$ of $X$; (ii) every quad of $T_{1} \cup T_{2}$ has a unique point in common with $X$. For every point $y$ of $\mathcal{S}$, we define $f(y)=\mathrm{d}(y, X)$. Then $f$ is a so-called semi-diagonal valuation of $\mathcal{S}$.

We take the following proposition from [15, Lemma 5.3]. 


\begin{tabular}{|c|c|c|c|c|c|}
\hline NEAR HEX. & SEMI-CL. & OVOID. & EXT. & SEMI-DIAG. & OTHER \\
\hline \hline $\mathbb{L}_{4} \times \mathbb{L}_{4} \times \mathbb{L}_{4}$ & 1 & 1 & 1 & - & - \\
\hline$W(3) \times \mathbb{L}_{4}$ & - & - & 1 & - & - \\
\hline$Q(4,3) \times \mathbb{L}_{4}$ & 1 & - & 2 & - & - \\
\hline $\mathrm{GQ}(3,5) \times \mathbb{L}_{4}$ & - & 1 & 2 & - & - \\
\hline$Q(5,3) \times \mathbb{L}_{4}$ & - & - & 1 & - & - \\
\hline$D W(5,3)$ & - & - & 1 & - & - \\
\hline$D Q(6,3)$ & - & - & - & - & - \\
\hline$D H(5,9)$ & - & - & - & - & - \\
\hline$Q(5,3) \otimes Q(5,3)$ & - & - & - & 1 & - \\
\hline GQ(3,5) $\otimes \mathrm{GQ}(3,5)$ & - & 1 & 1 & 1 & - \\
\hline Except, Type (I) & - & $?$ & - & - & - \\
\hline Except, Type (II) & - & $?$ & - & - & - \\
\hline Except, Type (III) & - & $?$ & - & - & - \\
\hline Except, Type (IV) & $?$ & $?$ & - & - & $?$ \\
\hline
\end{tabular}

Table 1: The non-classical valuations of the dense near hexagons of order $(3, t)$

Proposition 2.6 Let $F$ be a hex of a dense near octagon $\mathcal{S}$ and let $x$ be a point of $\mathcal{S}$ at distance 2 from $F$. For every point y of $F$, we define $f_{x}(y):=$ $d(x, y)-2$. Then $f_{x}$ is a valuation of $\mathcal{S}$ which is neither classical nor ovoidal.

In [12], we studied the valuations of the dense near hexagons of order $(3, t)$. The non-classical valuations of these near hexagons are given in Table 1 . We give the number of nonisomorphic valuations for each type. We put a question mark if nothing is known about their existence. The near hexagons $Q(4,3) \times \mathbb{L}_{4}$ and $\operatorname{GQ}(3,5) \times \mathbb{L}_{4}$ have two types of quads with ovoids, giving rise to two classes of extended valuations.

\section{The existence of big hexes}

In Theorem 1.5 of [12], we have shown the following:

Proposition 3.1 ([12]) Let $\mathcal{S}$ be a dense near octagon of order $(3, t)$ containing an exceptional hex $H$ of type $(I),(I I)$ or $(I I I)$, then $H$ is big in $\mathcal{S}$ and $\mathcal{S} \cong H \times \mathbb{L}_{4}$. 
In this section, we will prove that a dense near octagon $\mathcal{S}$ of order $(3, t)$ has big hexes if all hexes of $\mathcal{S}$ are classical or glued. The case of dense near octagons of order $(3, t)$ with an exceptional hex of type (IV) will be treated in Section 4.

Consider the following ordering of the known dense near hexagons of order $(3, t): \mathcal{N}_{1}=\mathbb{L}_{4} \times \mathbb{L}_{4} \times \mathbb{L}_{4}, \mathcal{N}_{2}=Q(4,3) \times \mathbb{L}_{4}, \mathcal{N}_{3}=W(3) \times \mathbb{L}_{4}, \mathcal{N}_{4}=$ $\operatorname{GQ}(3,5) \times \mathbb{L}_{4}, \mathcal{N}_{5}=Q(5,3) \times \mathbb{L}_{4}, \mathcal{N}_{6}=D W(5,3), \mathcal{N}_{7}=\operatorname{GQ}(3,5) \otimes \operatorname{GQ}(3,5)$, $\mathcal{N}_{8}=Q(5,3) \otimes Q(5,3), \mathcal{N}_{9}=D Q(6,3)$ and $\mathcal{N}_{10}=D H(5,9)$. In this section, we will prove the following result.

Proposition 3.2 Let $\mathcal{S}$ be a dense near octagon of order $(3, t)$ with the property that every hex is classical or glued. Let $i \in\{1, \ldots, 10\}$ be the biggest integer such that $\mathcal{S}$ contains a hex isomorphic to $\mathcal{N}_{i}$. Then every hex isomorphic to $\mathcal{N}_{i}$ is big in $\mathcal{S}$.

This proposition has the following corollary.

Corollary 3.3 Let $\mathcal{S}$ be a dense near octagon of order $(3, t)$ with the property that every hex is classical or glued. Then $\mathcal{S}$ contains a big hex and hence is isomorphic to one of the 28 near octagons mentioned in Proposition 1.1.

Suppose that Proposition 3.2 is not true. Then there exists a hex $H \cong \mathcal{N}_{i}$ and a point $x$ at distance 2 from $H$. The map $f: H \rightarrow \mathbb{N} ; y \mapsto \mathrm{d}(x, y)-2$ is a valuation of $H$ which is neither classical nor ovoidal by Proposition 2.6. Hence, $i \neq 9$ and $i \neq 10$ by Table 1 .

Lemma 3.4 If $i \leq 6$, then the valuation $f$ is semi-classical.

Proof. Suppose $f$ is not semi-classical, then $f$ is of extended type by Table 1. Let $Q$ denote the unique special quad of $H$ (with respect to $f$ ). Then $\langle x, Q\rangle$ is a hex, and its quad $Q$ is not big. This is impossible, since every hex of $\mathcal{S}$ is classical $(i \leq 6)$. Hence $f$ is semi-classical.

By Table $1, \mathcal{N}_{3}, \mathcal{N}_{4}, \mathcal{N}_{5}$ and $\mathcal{N}_{6}$ do not have semi-classical valuations. Hence,

Corollary $3.5 i \in\{1,2,7,8\}$.

The case $i=8$.

Lemma 3.6 If $Q$ is a special quad of $H$ (for the valuation $f$ ), then $\langle x, Q\rangle \cong$ $Q(5,3) \otimes Q(5,3)$. So, every quad through $x$ and a point of $O_{f}$ is a grid. 
Proof. If $Q$ is a special quad, then $\langle x, Q\rangle$ is a hex, and its grid-quad $Q$ is not big. It follows that $\langle x, Q\rangle$ is isomorphic to either $Q(5,3) \otimes Q(5,3)$ or $\mathrm{GQ}(3,5) \otimes \mathrm{GQ}(3,5)$. If $\langle x, Q\rangle \cong \mathrm{GQ}(3,5) \otimes \mathrm{GQ}(3,5)$, then there exists a GQ $(3,5)$-quad of $\langle x, Q\rangle$ intersecting a $Q(5,3)$-quad of $H$ in a line. The hex through these two quads would then contradict Proposition 2.4. So, $\langle x, Q\rangle$ is isomorphic to $Q(5,3) \otimes Q(5,3)$.

Now, let $y$ denote an arbitrary point of $O_{f}$. Since $f$ is a semi-diagonal valuation, see Table $1, y$ is contained in 9 special grid-quads $\mathcal{G}_{1}, \ldots, \mathcal{G}_{9}$. Let $L$ denote an arbitrary line through $y$ contained in the grid-quad $\langle x, y\rangle$ and let $L_{i}, i \in\{1, \ldots, 9\}$, be the line of $\mathcal{G}_{i}$ which is contained in a $Q(5,3)$-quad together with $L$. Two of these lines, say $L_{1}$ and $L_{2}$, are contained in the same $Q(5,3)$-quad of $H$. The hex $\left\langle L, L_{1}, L_{2}\right\rangle$ then has three $Q(5,3)$-quads through the point $y$, contradicting Proposition 2.4. (Recall that there are no $D H(5,9)$-hexes since $i=8$.)

\section{The case $i=7$.}

The valuation $f$ cannot be of extended type, otherwise $\langle x, Q\rangle$, with $Q$ the unique special quad of $f$, would be a hex containing a $\operatorname{GQ}(3,5)$-quad which is not big. This is impossible by Proposition 2.4. So, $f$ is of semi-diagonal type by Table 1 . If $Q$ is a special quad, then $\langle x, Q\rangle$ is a hex and its grid-quad $Q$ is not big. It follows that $\langle x, Q\rangle \cong \mathrm{GQ}(3,5) \otimes \mathrm{GQ}(3,5)$ (recall $i=7$ ).

We can now use a similar reasoning as in the case $i=8$ to obtain a contradiction. Let $y$ denote an arbitrary point of $O_{f}$ and let $\mathcal{G}_{1}, \ldots, \mathcal{G}_{5}$ denote the five special quads through $y$. Let $L$ denote a line through $y$ contained in the grid-quad $\langle x, y\rangle$ and let $L_{i}, i \in\{1, \ldots, 5\}$, denote the line of $\mathcal{G}_{i}$ which is contained in a $\operatorname{GQ}(3,5)$-quad with $L$. Two of these lines, say $L_{1}$ and $L_{2}$, are contained in the same GQ $(3,5)$-quad with $L$. The hex $\left\langle L, L_{1}, L_{2}\right\rangle$ has three GQ(3,5)-quads through the point $y$, contradicting Proposition 2.4.

\section{The case $i=2$.}

Every quad is isomorphic to $\mathbb{L}_{4} \times \mathbb{L}_{4}$ or $Q(4,3)$ and every hex is isomorphic to either $\mathbb{L}_{4} \times \mathbb{L}_{4} \times \mathbb{L}_{4}$ or $Q(4,3) \times \mathbb{L}_{4}$. Let $v$ denote the total number of points of $\mathcal{S}$ and let $n_{i}, i \in\{0, \ldots, 4\}$, denote the constants such that $\left|\Gamma_{i}(x)\right|=n_{i}$ for every point $x$ of $\mathcal{S}$ (see Proposition 2.1). We have $n_{0}=1, n_{1}=3(t+1)$ and $n_{0}-\frac{n_{1}}{3}+\frac{n_{2}}{9}-\frac{n_{3}}{27}+\frac{n_{4}}{81}=0([9$, Theorem 1.2]). Hence,

$$
v=n_{0}+n_{1}+n_{2}+n_{3}+n_{4}=4 n_{3}-8 n_{2}+28 n_{1}-80 n_{0} .
$$

By $[7$, Corollary $2+$ Section 6$]$ there exist constants $\alpha, \beta, A$ and $B$ such that each point is contained in $\alpha$ grid-quads, $\beta Q(4,3)$-quads, $A$ hexes isomorphic 
to $\mathbb{L}_{4} \times \mathbb{L}_{4} \times \mathbb{L}_{4}$ and $B$ hexes isomorphic to $Q(4,3) \times \mathbb{L}_{4}$. Since every two intersecting lines are contained in a unique quad, we have

$$
2 \alpha+12 \beta=(t+1) t .
$$

Since every two points at distance 2 , respectively distance 3 , are contained in a unique quad, respectively hex, we have

$$
\begin{aligned}
& n_{2}=9 \alpha+27 \beta, \\
& n_{3}=27 A+81 B .
\end{aligned}
$$

Counting in two different ways the number of pairs $(Q, L)$ with $Q$ a quad of order $(3, i), i \in\{1,3\}$, through $x$ and $L$ a line through $x$ not contained in $Q$, gives

$$
\begin{aligned}
& \beta(t-3)=B, \\
& \alpha(t-1)=3 A+12 B .
\end{aligned}
$$

By equations (1), (3), (4), (5) and (6), $n_{3}=9 \alpha(t-1)-27 \beta(t-3)$ and

$$
v=(36 t-108) \alpha+(108-108 t) \beta+84 t+4 .
$$

Since $H$ has no ovoids, we have $\Gamma_{3}(H)=\emptyset$.

Lemma 3.7 $\left|\Gamma_{2}(y) \cap H\right|=1$ for every point $y \in \Gamma_{2}(H)$.

Proof. For every point $z$ of $H$, define $g(z)=\mathrm{d}(y, z)-2$. Then $g$ is a valuation of $H$ which is neither classical nor ovoidal by Proposition 2.6.

Suppose $g$ is of extended type. If $Q$ is the unique special quad of $H$, then $\langle y, Q\rangle$ is a hex and its quad $Q$ is not big. This is impossible since all hexes are classical.

Hence, $g$ is semi-classical by Table 1 . If $z^{*}$ is the unique point of $H$ for which $g\left(z^{*}\right)=0$, then $\Gamma_{2}(y) \cap H=\left\{z^{*}\right\}$.

From $\left|\Gamma_{0}(H)\right|=160,\left|\Gamma_{1}(H)\right|=480(t-4),\left|\Gamma_{2}(H)\right|=160\left(n_{2}-63-45(t-4)\right)$ (use Lemma 3.7) and $\left|\Gamma_{3}(H)\right|=0$,

$$
v=1440 \alpha+4320 \beta-6720 t+16960 .
$$

By equations (2), (7) and (8), $\alpha$ and $\beta$ are solutions of the following linear system of equations:

$$
\left\{\begin{array}{l}
2 \alpha+12 \beta=t^{2}+t \\
(43-t) \cdot \alpha+(117+3 t) \cdot \beta=189 t-471
\end{array}\right.
$$

We find

$$
\alpha=\frac{3 t^{3}+120 t^{2}-2151 t+5652}{18 t-282}
$$


Lemma 3.8 Let $y$ be a point of $\mathcal{S}$ and let $Q$ be a quad. Then $y$ is classical with respect to $Q$ if and only if $d(y, Q) \leq 2$.

Proof. Obviously, $y$ is classical with respect to $Q$ if $\mathrm{d}(y, Q) \leq 1$ and $y$ is ovoidal with respect to $Q$ if $\mathrm{d}(y, Q)=3$. Suppose that $\mathrm{d}(y, Q)=2$ and that $y$ is ovoidal with respect to $Q$, then the quad $Q$ of the hex $\langle y, Q\rangle$ is not big, a contradiction.

Lemma 3.9 We have the following restrictions on the number $t+1$ :

(a) $t+1 \geq 4 \beta$;

(b) $t+1$ is even, $30 \leq t+1 \leq 50$ and $\frac{t+1}{2}-15 \leq \beta$.

Proof. (a) If $t+1<4 \beta$, then there exists a line $L$ which is contained in two $Q(4,3)$-quads $Q_{1}$ and $Q_{2}$. The hex $\left\langle Q_{1}, Q_{2}\right\rangle$ contains two $Q(4,3)$-quads through a line, contradicting $i=2$.

(b) Let $Q$ be a $Q(4,3)$-quad of $H$ not containing the unique point of $H$ at distance 2 from $x$. Let $\left\{x_{1}, \ldots, x_{10}\right\}$ denote the ovoid of $Q$ consisting of all points of $Q$ at distance 3 from $x$. Put $H_{i}:=\left\langle x, x_{i}\right\rangle, i \in\{1, \ldots, 10\}$. If $L$ is a line through $x$ contained in $\Gamma_{3}(Q)$, then the ovoids $\Gamma_{3}(z) \cap Q$, $z \in L$, of $Q$ partition the point set of $Q$. This is impossible, since $Q(4,3)$ has no partition in ovoids (see e.g. [4, Lemma 2.2]). So, every line through $x$ contains a point of $\Gamma_{2}(Q)$ and hence is contained in at least one of the hexes $H_{i}, i \in\{1, \ldots, 10\}$. Suppose that a line $L$ through $x$ is contained in two distinct hexes $H_{i_{1}}$ and $H_{i_{2}}\left(i_{1}, i_{2} \in\{1, \ldots, 10\}\right)$. If $y$ denotes the unique point of $L$ at distance 2 from $x_{i_{1}}$, then by Lemma 3.8,y is classical with respect to $Q$ and hence $\mathrm{d}\left(y, x_{i_{2}}\right)=\mathrm{d}\left(y, x_{i_{1}}\right)+\mathrm{d}\left(x_{i_{1}}, x_{i_{2}}\right)=4$, a contradiction, since $y$ and $x_{i_{2}}$ belong to $H_{i_{2}}$. Suppose that among the hexes $H_{1}, \ldots, H_{10}, \delta_{1}$ are isomorphic to $\mathbb{L}_{4} \times \mathbb{L}_{4} \times \mathbb{L}_{4}$ and $\delta_{2}$ are isomorphic to $Q(4,3) \times \mathbb{L}_{4}$. Then we have $\delta_{1}+\delta_{2}=10,3 \delta_{1}+5 \delta_{2}=t+1$ and $\delta_{2} \leq \beta$. Property (b) now easily follows.

Since $t \in\{29, \ldots, 49\}$ and $\alpha \in \mathbb{N}, t \in\{29,33,37,39,46\}$. The value $t=46$ is excluded since $t+1$ must be even. For $t=29$, we have $\alpha=489$ and $\beta=-9$. For $t=33$, we have $\alpha=555$ and $\beta=1$. For $t=37$, we have $\alpha=631$ and $\beta=12$. For $t=39$, we have $\alpha=672$ and $\beta=18$. None of these possibilities can occur by Lemma 3.9.

The case $i=1$.

In this case $\mathcal{S}$ is a so-called regular near octagon ([1, Section 6.4], [9, Chapter $3]$ ) with parameters $s=3, t_{2}=1, t_{3}=2$ and $t$. Let $A$ denote the collinearity 
matrix of $A$. There are well-known techniques for calculating the eigenvalues and multiplicities of $A$ in function of $t$, see [1], [9], [18]. (In [9, Section 3.3] or [18, Theorem 7.8], there are explicit formulas for the eigenvalues and multiplicities in function of the parameters.) There exists only one value of $t$ for which all eigenvalues are integral, namely $t=3$. In this case $\mathcal{S} \cong$ $\mathbb{L}_{4} \times \mathbb{L}_{4} \times \mathbb{L}_{4} \times \mathbb{L}_{4}$. But this is impossible since we assumed that there exists a point $x$ and a hex $H$ such that $\mathrm{d}(x, H)=2$.

\section{Dense near octagons of order $(3, t)$ without big hexes}

In this section, we will prove Theorem 1.2. So, let $\mathcal{S}$ denote a dense near octagon of order $(3, t)$ without big hexes. By Proposition 3.1,

Lemma $4.1 \mathcal{S}$ does not contain exceptional hexes of type $(I),(I I)$ or $(I I I)$.

By Corollary 3.3, $\mathcal{S}$ has a hex $H$ of type (IV). By Proposition 2.1, there exist constants $n_{i}, i \in\{0, \ldots, 4\}$, such that $\left|\Gamma_{i}(x)\right|=n_{i}$ for every point $x$ of $\mathcal{S}$. Obviously, $n_{0}=1$ and $n_{1}=3(t+1)$.

Lemma 4.2 Every quad $Q$ isomorphic to either $W(3), \operatorname{GQ}(3,5)$ or $Q(5,3)$ is classical in $\mathcal{S}$.

Proof. By Proposition 2.2, this holds if $Q \cong W(3)$ or $Q \cong Q(5,3)$, since these generalized quadrangles do not have ovoids [17, 3.4.1]. So, Suppose $Q \cong \mathrm{GQ}(3,5)$. Let $x$ denote an arbitrary point of $\mathcal{S}$. If $\mathrm{d}(x, Q) \leq 1$, then $x$ is obviously classical with respect to $Q$.

Suppose $\mathrm{d}(x, Q)=2$ and that $x$ is ovoidal with respect to $Q$, then the hex $\langle x, Q\rangle$ contains a quad isomorphic to $\mathrm{GQ}(3,5)$ which is not big, contradicting Proposition 2.4. Hence, every point of $\Gamma_{2}(Q)$ is classical with respect to $Q$.

Suppose now that $x \in \Gamma_{3}(Q)$. Then $x$ is ovoidal with respect to $Q$. Let $x^{\prime}$ be a point collinear with $x$ at distance 2 from $Q$. Then $x^{\prime}$ is classical with respect to $Q$. Now, let $L$ be the line $x^{\prime} x$. By Lemma 9 of [2], every point of $L \backslash\left\{x^{\prime}\right\}$ is ovoidal with respect to $Q$. The three ovoids $\Gamma_{3}(z) \cap Q, z \in L \backslash\left\{x^{\prime}\right\}$, all contain the point $\pi_{Q}\left(x^{\prime}\right)$ and partition the set of points of $Q$ at distance 2 from $\pi_{Q}\left(x^{\prime}\right)$. Such a configuration of ovoids cannot exist in a $\mathrm{GQ}(3,5)$-quad, see [4, Lemma 2.2]. This proves the lemma.

Lemma 4.3 If a certain $Q(4,3)$-quad is classical in $\mathcal{S}$, then all $Q(4,3)$-quads are classical in $\mathcal{S}$. 
Proof. Let $Q$ be a given $Q(4,3)$-quad. If $x$ is a point of $\Gamma_{2}(Q)$ which is ovoidal with respect to $Q$, then the hex $\langle x, Q\rangle$ contains a $Q(4,3)$-quad which is not big. By Proposition 2.4, $\langle x, Q\rangle$ is an exceptional hex of type (I), (II) or (III). This contradicts Lemma 4.1. Hence, every point of $\Gamma_{2}(Q)$ is classical with respect to $Q$. Obviously, every point of $\Gamma_{3}(Q)$ is ovoidal with respect to $Q$. Hence, a $Q(4,3)$-quad $Q$ is classical in $\mathcal{S}$ if and only if the total number of points of $\mathcal{S}$ is equal to $\left|\Gamma_{0}(Q)\right|+\left|\Gamma_{1}(Q)\right|+\left|\Gamma_{2}(Q)\right|$. Now, $\left|\Gamma_{0}(Q)\right|=40$, $\left|\Gamma_{1}(Q)\right|=40 \cdot 3(t-3)$ and $\left|\Gamma_{2}(Q)\right|=|Q| \cdot\left(n_{2}-27-12 \cdot 3(t-3)\right)$. Hence, $\left|\Gamma_{0}(Q)\right|+\left|\Gamma_{1}(Q)\right|+\left|\Gamma_{2}(Q)\right|$ is independent from the chosen $Q(4,3)$-quad $Q$. The lemma now readily follows.

The following lemma is a special case of Theorem 2.32 of [9].

Lemma 4.4 Let $Q$ be a quad of $\mathcal{S}$ which is classical in $\mathcal{S}$, then every hex which meets $Q$ has at least one line in common with $Q$.

Lemma 4.5 No hex is isomorphic to $D W(5,3)$.

Proof. Suppose the contrary. Let $H$ be a hex of $\mathcal{S}$ isomorphic to $D W(5,3)$. Since $H$ is not big in $\mathcal{S}$, there exists a point $x$ in $\mathcal{S}$ at distance 2 from $H$. The map $f_{x}: H \rightarrow \mathbb{N} ; y \mapsto \mathrm{d}(x, y)-2$ is a non-classical and non-ovoidal valuation by Proposition 2.6. By Table $1, f_{x}$ is of extended type. If $Q$ is the unique special quad, then the hex $\langle x, Q\rangle$ contains a $Q(4,3)$-quad which is not big, contradicting Proposition 2.4 and Lemma 4.1.

Lemma 4.6 If $Q$ is a quad of $\mathcal{S}$, then every point $x$ of $\mathcal{S}$ is contained in a quad isomorphic to $Q$.

Proof. By connectedness of $\mathcal{S}$, it suffices to prove this for every point $x \in \Gamma_{1}(Q)$. Now, consider the hex $\langle x, Q\rangle$ and apply Proposition 2.4.

Lemma 4.7 Precisely one of the following cases occurs:

(1) Every quad is isomorphic to $\mathbb{L}_{4} \times \mathbb{L}_{4}$ and every hex is isomorphic to either $\mathbb{L}_{4} \times \mathbb{L}_{4} \times \mathbb{L}_{4}$ or an exceptional near hexagon of type $(I V)$.

(2) Every quad is isomorphic to either $\mathbb{L}_{4} \times \mathbb{L}_{4}$ or $Q(4,3)$ and both types of quads occur. Every hex is isomorphic to either $\mathbb{L}_{4} \times \mathbb{L}_{4} \times \mathbb{L}_{4}, Q(4,3) \times$ $\mathbb{L}_{4}$ or an exceptional near hexagon of type $(I V)$. No Q $(4,3)$-quad is classical.

Proof. Let $\mathcal{Q}$ denote the set of all thick quads $Q$ (i.e. $Q \neq \mathbb{L}_{4} \times \mathbb{L}_{4}$ ) which are classical in $\mathcal{S}$. If $\mathcal{Q} \neq \emptyset$, then by Proposition 2.3 and Lemmas 4.2, 4.3, 4.6, there exists a quad $Q \in \mathcal{Q}$ containing a point $x \in H$. By Lemma 4.4, 
$Q \cap H$ is a line $L$. If every line through $x$ is contained in $Q \cup H$, then by Theorem 2.31 of [9], $H$ is big in $\mathcal{S}$, which is impossible. Hence, there exists a line $L^{\prime}$ through $x$ not contained in $Q \cup H$. Let $Q^{\prime}$ denote a quad through $L^{\prime}$ such that $Q \cap Q^{\prime}=\{x\}$. Now, by Lemma 4.4, there are precisely $t_{Q}+1 \leq 10$ hexes through $Q^{\prime}$. On the other hand, every hex through $Q^{\prime}$ intersects $H$ in at most a quad. So, if $Q^{\prime} \cap H=\{x\}$, then $t_{Q}+1 \geq \frac{t_{H}+1}{2}=\frac{35}{2}$, a contradiction. If $Q^{\prime} \cap H$ is a line, then $t_{Q}+1 \geq t_{H}$, also a contradiction. As a consequence, $\mathcal{Q}=\emptyset$. Hence by Lemma $4.2, \mathcal{S}$ does not contain quads isomorphic to $W(3)$, $Q(5,3)$ or $\mathrm{GQ}(3,5)$. Also no $Q(4,3)$-quad is classical in $\mathcal{S}$. The lemma now readily follows.

We will now show that case (2) in Lemma 4.7 cannot occur. Suppose the contrary and let $\mathcal{S}$ satisfy the conditions of Lemma 4.7 (2). Then we can say the following.

Lemma 4.8 There exist constants $\alpha_{1}, \alpha_{2}, \beta_{1}, \beta_{2}$ and $\beta_{3}$ such that every point of $\mathcal{S}$ is contained in $\alpha_{1} \mathbb{L}_{4} \times \mathbb{L}_{4}$-quads, $\alpha_{2} Q(4,3)$-quads, $\beta_{1} \mathbb{L}_{4} \times \mathbb{L}_{4} \times \mathbb{L}_{4^{-}}$hexes, $\beta_{2} Q(4,3) \times \mathbb{L}_{4}$-hexes and $\beta_{3}$ exceptional hexes of type $(I V)$.

Proof. This has been shown in [7] (case (5) of Section 6).

Lemma 4.9 There exist constants $\gamma_{1}, \gamma_{2}, \gamma_{3}$ such that for every point $x$ and every $Q(4,3)$-quad $Q$ such that $d(x, Q)=3$, there are $\gamma_{1} \mathbb{L}_{4} \times \mathbb{L}_{4} \times \mathbb{L}_{4}$-hexes, $\gamma_{2} Q(4,3) \times \mathbb{L}_{4}$-hexes and $\gamma_{3}$ exceptional hexes through $x$ meeting $Q$ in a point.

Proof. Let $O$ be the ovoid $\Gamma_{3}(x) \cap Q$ of $Q$. Since $|O|=10$, there are precisely 10 hexes through $x$ which meet $Q$ in a point. Hence,

$$
\gamma_{1}+\gamma_{2}+\gamma_{3}=10 \text {. }
$$

We will now show that every line $L$ through $x$ is contained in precisely one of the 10 above-mentioned hexes. If $L \subseteq \Gamma_{3}(Q)$, then the ovoids $\Gamma_{3}(z) \cap Q$, $z \in L$, of $Q$ determine a partition of $Q$ in ovoids, which is impossible, since $Q(4,3)$ does not have a partition in ovoids [4, Lemma 2.2]. Hence, $L$ contains a point $z^{\prime} \in \Gamma_{2}(Q)$. If $z^{\prime}$ is ovoidal with respect to $Q$, then the hex $\left\langle z^{\prime}, Q\right\rangle$ contains a $Q(4,3)$-quad which is not big, a contradiction. Hence, $z^{\prime}$ is classical with respect to $Q$. Obviously, the line $L$ is contained in the hex $\left\langle x, \pi_{Q}\left(z^{\prime}\right)\right\rangle$. Suppose that $L$ is contained in another hex $H^{\prime}$ which meets $Q$ in a point $u$. Then $\mathrm{d}\left(z^{\prime}, u\right)=\mathrm{d}\left(z^{\prime}, \pi_{Q}\left(z^{\prime}\right)\right)+\mathrm{d}\left(\pi_{Q}\left(z^{\prime}\right), u\right)=2+2=4$, contradicting the fact that $z^{\prime}$ and $u$ belong to the hex $H^{\prime}$. Hence, every line through $x$ is contained in precisely one of the hexes through $x$ meeting $Q$. It follows that

$$
3 \gamma_{1}+5 \gamma_{2}+35 \gamma_{3}=t+1
$$


By (9) and (10), $\frac{\gamma_{2}}{16}+\gamma_{3}=\frac{t-29}{32}$. Hence,

$$
\frac{t-49}{32} \leq \gamma_{3} \leq \frac{t-29}{32}
$$

since $\gamma_{2} \leq 10$. Now, $\gamma_{3}$ is uniquely determined by (11) and hence only depends on $t$. By (9) and (10), also $\gamma_{1}$ and $\gamma_{2}$ only depend on $t$. This proves the lemma.

Lemma 4.10 (a) Let $x$ be a point and let $Q$ be a $Q(4,3)$-quad such that $d(x, Q)=3$. Then every exceptional hex through $x$ meets $Q$.

(b) $t+1 \geq 62$.

Proof. (a) Let $H_{1}, \ldots, H_{10}$ denote the 10 hexes through $x$ meeting $Q$. Suppose that $H^{\prime}$ is an exceptional hex through $x$ disjoint from $Q$. Then $H^{\prime}$ meets each $H_{i}, i \in\{1, \ldots, 10\}$, in at most a quad. Hence, at most 20 lines of $H^{\prime}$ through $x$ are contained in $H_{1} \cup \cdots \cup H_{10}$. This is impossible, since $t_{H^{\prime}}+1=35$ and since every line through $x$ is contained in $H_{1} \cup \cdots \cup H_{10}$, by the proof of Lemma 4.9.

(b) By equation (10), $t+1=3\left(\gamma_{1}+\gamma_{2}+\gamma_{3}\right)+2 \gamma_{2}+32 \gamma_{3}$. Now, $\gamma_{3} \geq 1$ (see (a) and Lemma 4.8), $\gamma_{2} \geq 0$ and $\gamma_{1}+\gamma_{2}+\gamma_{3}=10$. Hence, $t+1 \geq 62$.

Lemma 4.11 There exists a constant $\delta$ such that for every point $x$ of $\mathcal{S}$, there are precisely $\delta Q(4,3)$-quads $Q$ for which $d(x, Q)=3$.

Proof. Let $x$ be a given point of $\mathcal{S}$. Let $N_{i}, i \in\{0,1,2,3\}$, denote the number of $Q(4,3)$-quads such that $\mathrm{d}(x, Q)=i$. Then $N_{0}+N_{1}+N_{2}+N_{3}$ is the total number of $Q(4,3)$-quads of $\mathcal{S}$. If $\mathrm{d}(x, Q) \leq 2$, then $x$ is classical with respect to $Q$ and hence $Q$ contains a unique point nearest to $x$. We have (with $\alpha_{2}$ as in Lemma 4.8)

$$
\begin{aligned}
\alpha_{2} & =N_{0}, \\
3(t+1) \cdot \alpha_{2} & =N_{1}+12 N_{0}, \\
n_{2} \cdot \alpha_{2} & =N_{2}+12 N_{1}+27 N_{0} .
\end{aligned}
$$

By (12), (13) and (14), $N_{0}, N_{1}$ and $N_{2}$ are independent from the chosen point $x$. Hence, also $N_{3}$ does not depend on the chosen point $x$.

Remark. Since $\Gamma_{3}(Q) \neq \emptyset$ for every $Q(4,3)$-quad $Q, \delta \geq 1$.

Lemma 4.12 Let $x$ be a point of $\mathcal{S}$. Then there are precisely $\beta_{3}=\gamma_{3}$ exceptional hexes through $x$ and any two of these hexes intersect each other only in the point $x$. 
Proof. Since $\delta \geq 1$, there exists a $Q(4,3)$-quad $Q$ such that $\mathrm{d}(x, Q)=3$. Now, the lemma follows from Lemma 4.10 and the proof of Lemma 4.9.

We are now ready to derive a contradiction. Let $Q$ be a $Q(4,3)$-quad, let $x$ be a point of $Q$ and let $G$ be a grid-quad through $x$ such that $G \cap Q=\{x\}$. (Such a grid exists through every exceptional hex through $x$.) Let $y$ be a point of $G$ at distance 2 from $x$. Let $H_{1}, \ldots, H_{t-5}$ denote all the $t-5 Q(4,3) \times \mathbb{L}_{4}$-hexes through $Q$ which intersect $G$ only in the point $x$. Then $\mathrm{d}\left(y, H_{i}\right)=2$ for every $i \in\{1, \ldots, t-5\}$. (If $y^{\prime}$ were a point of $H_{i}$ collinear with $y$, then $y^{\prime}$ is on a shortest path between $y$ and $x$ and hence $y^{\prime} \in G$, which is impossible.) Put $f_{i}: H_{i} \rightarrow \mathbb{N} ; u \mapsto \mathrm{d}(y, u)-2$. By Proposition 2.6, $f_{i}$ is a valuation of $H_{i}$ which is neither classical nor ovoidal.

Suppose that $f_{i}$ is semi-classical for a certain $i \in\{1, \ldots, t-5\}$. Let $R_{1}, R_{2}, R_{3}$ denote the three $Q(4,3)$-quads of $H_{i}$ different from $Q$ and let $u_{i}, i \in\{1,2,3\}$, denote the unique point of $R_{i}$ collinear with $x$. Then $\mathrm{d}\left(y, R_{1}\right)=\mathrm{d}\left(y, R_{2}\right)=$ $\mathrm{d}\left(y, R_{3}\right)=3$. For each $i \in\{1,2,3\}$, the $\gamma_{3}$ exceptional hexes through $y$ are precisely the $\gamma_{3}$ exceptional hexes through $y$ intersecting $R_{i}$. If $\left\langle y, u_{1}\right\rangle=$ $\left\langle y, u_{2}\right\rangle=\left\langle y, u_{3}\right\rangle$ is not an exceptional hex, then there are at least $3 \gamma_{3}$ exceptional hexes through $y$ intersecting $H_{i}$, a contradiction. Hence, $\left\langle y, u_{1}\right\rangle=$ $\left\langle y, u_{2}\right\rangle=\left\langle y, u_{3}\right\rangle$ is an exceptional hex and there are at least $3 \gamma_{3}-2$ exceptional hexes through $y$ intersecting $H_{i}$. It follows that $\gamma_{3}=1$. Since $t+1 \geq 62$, there exists a line $L$ through $x$ not contained in $\left\langle y, u_{1}\right\rangle \cup Q$. Let $j \in\{1, \ldots, t-5\}$ such that $H_{j}=\langle Q, L\rangle$. By the above reasoning we know that $f_{j}$ cannot be semi-classical (otherwise $\langle y, L\rangle$ and $\left\langle y, u_{1}\right\rangle$ are two distinct exceptional hexes through $y$, contradicting $\gamma_{3}=1$ ). Hence, there exists a special quad $Q^{\prime}$ in $H_{j}$. But then the hex $\left\langle y, Q^{\prime}\right\rangle$ must be exceptional since its quad $Q^{\prime}$ is not big. Again this is impossible, since $\left\langle y, u_{1}\right\rangle$ is the only exceptional hex through $y$.

Hence, all valuations $f_{i}, i \in\{1, \ldots, t-5\}$, are of extended type. If $Q^{\prime}$ is a special quad with respect to one of these valuations, then $Q^{\prime} \cong \mathbb{L}_{4} \times \mathbb{L}_{4}$ and $\left\langle y, Q^{\prime}\right\rangle$ is exceptional, since the quad $Q^{\prime}$ of the hex $\left\langle y, Q^{\prime}\right\rangle$ is not big. In fact $H^{*}:=\left\langle y, Q^{\prime}\right\rangle$ is the unique exceptional hex through the grid $G$, see Lemma 4.12. It follows that each of the $t-5$ hexes $H_{1}, \ldots, H_{t-5}$ meet the unique exceptional hex through $G$. This means that every line through $x$ not contained in $Q$ is contained in $H^{*}$. This is impossible, since $t+1 \geq 62$.

We can conclude that case (2) of Lemma 4.7 cannot occur. This proves Theorem 1.2. 


\section{Corollaries}

\subsection{Proof of Theorem 1.4}

For every point $x$ of $\mathcal{S}$, let $\alpha(x)$ denote the number of convex subpolygons through $x$ isomorphic to an element of $\mathcal{V}$.

Now, let $x_{1}$ and $x_{2}$ denote two distinct collinear points of $\mathcal{S}$. For every convex subpolygon $F$ of $\mathcal{S}$ through the line $x_{1} x_{2}$, let $\mu_{F}$ denote the number of convex sub- $2 \delta$-gons of $F$ through $x_{1} x_{2}$ isomorphic to an element of $\mathcal{V}$. Since every sub- $2 \delta$-gon through $x_{1}$ not containing $x_{2}$ is contained in a unique sub- $(2 \delta+2)$-gon containing $x_{2}$, we have

$$
\alpha\left(x_{1}\right)=\mu_{\mathcal{S}}+\sum_{F}\left(\alpha_{F}-\mu_{F}\right),
$$

where the summation ranges over all convex sub- $(2 \delta+2)$-gons $F$ through the line $x_{1} x_{2}$. Similarly,

$$
\alpha\left(x_{2}\right)=\mu_{\mathcal{S}}+\sum_{F}\left(\alpha_{F}-\mu_{F}\right) .
$$

Hence, $\alpha\left(x_{1}\right)=\alpha\left(x_{2}\right)$ for any two distinct collinear points $x_{1}$ and $x_{2}$ of $\mathcal{S}$. The lemma now follows from the connectedness of $\mathcal{S}$.

\subsection{Applications}

In Table 2, we list the number of quads of each type through a given point of a dense near hexagon of order $(3, t)$. We have used the notation $\otimes^{2} \mathrm{GQ}(3,5)$ for the glued near hexagon $\mathrm{GQ}(3,5) \otimes \mathrm{GQ}(3,5)$ and the notation $\otimes^{2} Q(5,3)$ for the glued near hexagon $Q(5,3) \otimes Q(5,3)$. Corollary 1.5 is an immediate corollary of Theorem 1.4 and Table 2 .

In Table 3, we list the number of hexes of each type through a given point of a known dense near octagon of order $(3, t)$. In the table, $\mu_{i}, i \in$ $\{1, \ldots, 10\}$, denotes the number of $\mathcal{H}_{i}$-hexes through a given point of the near octagon. For the definition of $\mathcal{H}_{i}$, see Table 2. The numbers in the table are readily deduced from [6] and [8]. (In Section 3 of [6], we have studied the convex subpolygons of product and glued near polygons. For the glued near octagons of type $\otimes^{3} \mathrm{GQ}(3,5)$ and $\otimes^{3} Q(5,3)$, we also need the information provided by Propositions 5.1, 5.2 and 5.3 of [8].) Notice that only $\left(\otimes^{2} Q(5,3)\right) \otimes_{2} Q(5,3)$ has two types of points. There are points which are contained in $729 \mathbb{L}_{4} \times \mathbb{L}_{4} \times \mathbb{L}_{4}$-hexes and $3 \otimes^{2} Q(5,3)$-hexes, and points which are contained in $648 \mathbb{L}_{4} \times \mathbb{L}_{4} \times \mathbb{L}_{4}$-hexes, $18 Q(5,3) \times \mathbb{L}_{4}$ hexes and 2 $\otimes^{2} Q(5,3)$-hexes. Corollaries 1.6 and 1.7 now readily follow from Proposition 1.1, Theorem 1.2, Corollary 1.3, Theorem 1.4 and Table 3. 


\begin{tabular}{|l|c|c|c|c|c|}
\hline & $\mathbb{L}_{4} \times \mathbb{L}_{4}$ & $\mathrm{~W}(3)$ & $\mathrm{Q}(4,3)$ & $\mathrm{GQ}(3,5)$ & $Q(5,3)$ \\
\hline $\mathcal{H}_{1}=\mathbb{L}_{4} \times \mathbb{L}_{4} \times \mathbb{L}_{4}$ & 3 & - & - & - & - \\
\hline $\mathcal{H}_{2}=W(3) \times \mathbb{L}_{4}$ & 4 & 1 & - & - & - \\
\hline $\mathcal{H}_{3}=Q(4,3) \times \mathbb{L}_{4}$ & 4 & - & 1 & - & - \\
\hline $\mathcal{H}_{4}=\mathrm{GQ}(3,5) \times \mathbb{L}_{4}$ & 6 & - & - & 1 & - \\
\hline $\mathcal{H}_{5}=Q(5,3) \times \mathbb{L}_{4}$ & 10 & - & - & - & 1 \\
\hline $\mathcal{H}_{6}=D W(5,3)$ & - & - & 13 & - & - \\
\hline $\mathcal{H}_{7}=D Q(6,3)$ & - & 13 & - & - & - \\
\hline $\mathcal{H}_{8}=D H(5,9)$ & - & - & - & 91 & - \\
\hline $\mathcal{H}_{9}=\otimes^{2} \mathrm{GQ}(3,5)$ & 25 & - & - & 2 & - \\
\hline $\mathcal{H}_{10}=\otimes^{2} Q(5,3)$ & 81 & - & - & - & 2 \\
\hline Exceptional, type I & 160 & - & 5 & - & - \\
\hline Exceptional, type II & 171 & - & 10 & - & - \\
\hline Exceptional, type III & 120 & - & 43 & - & - \\
\hline Exceptional, type IV & 595 & - & - & - & - \\
\hline
\end{tabular}

Table 2: The number of quads through a given point of a dense near hexagon of order $(3, t)$

\section{Acknowledgment}

The author is a Postdoctoral Fellow of the Research Foundation - Flanders.

\section{References}

[1] A. E. Brouwer, A. M. Cohen and A. Neumaier. Distance-regular graphs. Springer-Verlag, 1989.

[2] A. E. Brouwer and H. A. Wilbrink. The structure of near polygons with quads. Geom. Dedicata 14 (1983), 145-176.

[3] P. J. Cameron. Dual polar spaces. Geom. Dedicata 12 (1982), 75-86.

[4] B. De Bruyn. Near hexagons with four points on a line. Adv. Geom. 1 (2001), 211-228.

[5] B. De Bruyn. The glueing of near polygons. Bull. Belg. Math. Soc. Simon Stevin 9 (2002), 621-630.

[6] B. De Bruyn. Decomposable near polygons. Ann. Comb. 8 (2004), 251267. 


\begin{tabular}{|c|c|c|c|c|c|c|c|c|c|c|}
\hline & $\mu_{1}$ & $\mu_{2}$ & $\mu_{3}$ & $\mu_{4}$ & $\mu_{5}$ & $\mu_{6}$ & $\mu_{7}$ & $\mu_{8}$ & $\mu_{9}$ & $\mu_{10}$ \\
\hline $\mathbb{L}_{4} \times \mathbb{L}_{4} \times \mathbb{L}_{4} \times \mathbb{L}_{4}$ & 4 & - & - & - & - & - & - & - & - & - \\
\hline $\mathbb{L}_{4} \times \mathbb{L}_{4} \times W(3)$ & 4 & 2 & - & - & - & - & - & - & - & - \\
\hline $\mathbb{L}_{4} \times \mathbb{L}_{4} \times Q(4,3)$ & 4 & - & 2 & - & - & - & - & - & - & - \\
\hline $\mathbb{L}_{4} \times \mathbb{L}_{4} \times \mathrm{GQ}(3,5)$ & 6 & - & - & 2 & - & - & - & - & - & - \\
\hline $\mathbb{L}_{4} \times \mathbb{L}_{4} \times Q(5,3)$ & 10 & - & - & - & 2 & - & - & - & - & - \\
\hline $\mathbb{L}_{4} \times D W(5,3)$ & - & - & 13 & - & - & 1 & - & - & - & - \\
\hline $\mathbb{L}_{4} \times D Q(6,3)$ & - & 13 & - & - & - & - & 1 & - & - & - \\
\hline $\mathbb{L}_{4} \times D H(5,9)$ & - & - & - & - & 91 & - & - & 1 & - & - \\
\hline $\mathbb{L}_{4} \times \otimes^{2} \mathrm{GQ}(3,5)$ & 25 & - & - & 2 & - & - & - & - & 1 & - \\
\hline $\mathbb{L}_{4} \times \otimes^{2} Q(5,3)$ & 81 & - & - & - & 2 & - & - & - & - & 1 \\
\hline$W(3) \times W(3)$ & - & 8 & - & - & - & - & - & - & - & - \\
\hline$W(3) \times Q(4,3)$ & - & 4 & 4 & - & - & - & - & - & - & - \\
\hline$W(3) \times \mathrm{GQ}(3,5)$ & - & 6 & - & 4 & - & - & - & - & - & - \\
\hline$W(3) \times Q(5,3)$ & - & 10 & - & - & 4 & - & - & - & - & - \\
\hline$Q(4,3) \times Q(4,3)$ & - & - & 8 & - & - & - & - & - & - & - \\
\hline$Q(4,3) \times \mathrm{GQ}(3,5)$ & - & - & 6 & 4 & - & - & - & - & - & - \\
\hline$Q(4,3) \times Q(5,3)$ & - & - & 10 & - & 4 & - & - & - & - & - \\
\hline $\mathrm{GQ}(3,5) \times \mathrm{GQ}(3,5)$ & - & - & - & 12 & - & - & - & - & - & - \\
\hline $\operatorname{GQ}(3,5) \times Q(5,3)$ & - & - & - & 10 & 6 & - & - & - & - & - \\
\hline$Q(5,3) \times Q(5,3)$ & - & - & - & - & 20 & - & - & - & - & - \\
\hline$D W(7,3)$ & - & - & - & - & - & 40 & - & - & - & - \\
\hline$D Q(8,3)$ & - & - & - & - & - & - & 40 & - & - & - \\
\hline$D H(7,9)$ & - & - & - & - & - & - & - & 820 & - & - \\
\hline$D H(5,9) \otimes Q(5,3)$ & - & - & - & - & 729 & - & - & 1 & - & 10 \\
\hline$\left(\otimes^{2} \mathrm{GQ}(3,5)\right) \otimes_{1} \mathrm{GQ}(3,5)$ & 125 & - & - & - & - & - & - & - & 3 & - \\
\hline$\left(\otimes^{2} Q(5,3)\right) \otimes_{1} Q(5,3)$ & 729 & - & - & - & - & - & - & - & - & 3 \\
\hline$\left(\otimes^{2} \mathrm{GQ}(3,5)\right) \otimes_{2} \mathrm{GQ}(3,5)$ & 100 & - & - & 10 & - & - & - & - & 2 & - \\
\hline$\left(\otimes^{2} Q(5,3)\right) \otimes_{2} Q(5,3)$ & $\begin{array}{l}729 \\
648\end{array}$ & $\begin{array}{l}- \\
-\end{array}$ & $\begin{array}{l}- \\
-\end{array}$ & $\begin{array}{l}- \\
-\end{array}$ & $\begin{array}{l}- \\
18\end{array}$ & $\begin{array}{l}- \\
-\end{array}$ & $\begin{array}{l}- \\
-\end{array}$ & $\begin{array}{l}- \\
-\end{array}$ & $\begin{array}{l}- \\
-\end{array}$ & $\begin{array}{l}3 \\
2\end{array}$ \\
\hline
\end{tabular}

Table 3: The number of hexes through a given point of a known dense near octagon of order $(3, t)$ 
[7] B. De Bruyn. Dense near polygons with two types of quads and three types of hexes. J. Combin. Math. Combin. Comput. 57 (2006), 113-128.

[8] B. De Bruyn. Compatible spreads of symmetry in near polygons. J. Algebraic Combin. 23 (2006), 137-148.

[9] B. De Bruyn. Near polygons. Frontiers in Mathematics. Birkhäuser Verlag, Basel, 2006.

[10] B. De Bruyn. Valuations of glued near hexagons. J. Combin. Des. 15 (2007), 35-48.

[11] B. De Bruyn. Dense near octagons with four points on each line, I. Ann. Comb. 11 (2007), 127-142.

[12] B. De Bruyn. Dense near octagons with four points on each line, II. Adv. Geom. 7 (2007), 191-206.

[13] B. De Bruyn and P. Vandecasteele. Valuations of near polygons. Glasg. Math. J. 47 (2005), 347-361.

[14] B. De Bruyn and P. Vandecasteele. The valuations of the near hexagons related to the Witt-designs $S(5,6,12)$ and $S(5,8,24)$. J. Combin. Des. 14 (2006), 214-228.

[15] B. De Bruyn and P. Vandecasteele. The classification of the slim dense near octagons. European J. Combin. 28 (2007), 410-428.

[16] S. Dixmier and F. Zara. Etude d'un quadrangle généralisé autour de deux de ses points non liés. preprint 1976.

[17] S. E. Payne and J. A. Thas. Finite Generalized Quadrangles. Volume 110 of Research Notes in Mathematics. Pitman, Boston, 1984.

[18] S. A. Shad and E. E. Shult. The near $n$-gon geometries. preprint 1979.

[19] E. E. Shult and A. Yanushka. Near $n$-gons and line systems. Geom. Dedicata 9 (1980), 1-72. 